\title{
Development of Tilt Control System Using Electro-Hydraulic Actuators
}

Mamoru ENOMOTO

Senior Researcher,

Running Gear Lab., Vehicle Structure Technology Div.
Masako KAMIYAMA, Ph.D.

Senior Researcher,

Track Geometry \& Maintenance Lab.,

Track Technology Div.

Toshihiro HAMADA

Former Researcher,

Running Gear Lab., Vehicle Structure Technology Div.

\section{Shogo KAMOSHITA}

Senior Researcher,

Vehicle Noise \& Vibration Lab.,

Kimiaki SASAKI

Senior Researcher,

Vehicle Noise \& Vibration Lab.,

Vehicle Structure Technology Div.

\section{Akihito KAZATO}

Researcher,

To improve the performance and riding comfort of tilt-controlled vehicles, we developed a next-generation tilt control system that uses a position detecting system, a novel tilt pattern, high performance tilt actuators and other new technical elements. Under this system, the running train position is detected by curvature collation based on GPS signals and the tilting angle target pattern calculated to optimize the riding comfort evaluation index. The pattern thus created is called the JT pattern, according to which the tilt actuators are electro-hydraulically powered. Running tests have proved that low-frequency vibration causes train motion sickness has decreased as a result of this system.

Keywords: tilting, EHA, JT pattern, motion sickness, riding comfort

\section{Introduction}

Vehicles equipped with tilt control systems, which have been in operational use in Japan since 1989, play an important role in high-speed running on routes with many curves in mountainous areas and along irregular coastlines. All Japan Railway (JR) passenger companies now utilize these vehicles on intercity limited express services.

As shown in Fig. 1, the conventional control system firstly finds the train's current position by detecting an automatic train stop (ATS) ground unit on the track and the number of pulse signals generated by the wheel rotations. Secondly, this system drives the tilt actuator installed in the bogie in order to tilt the body according to

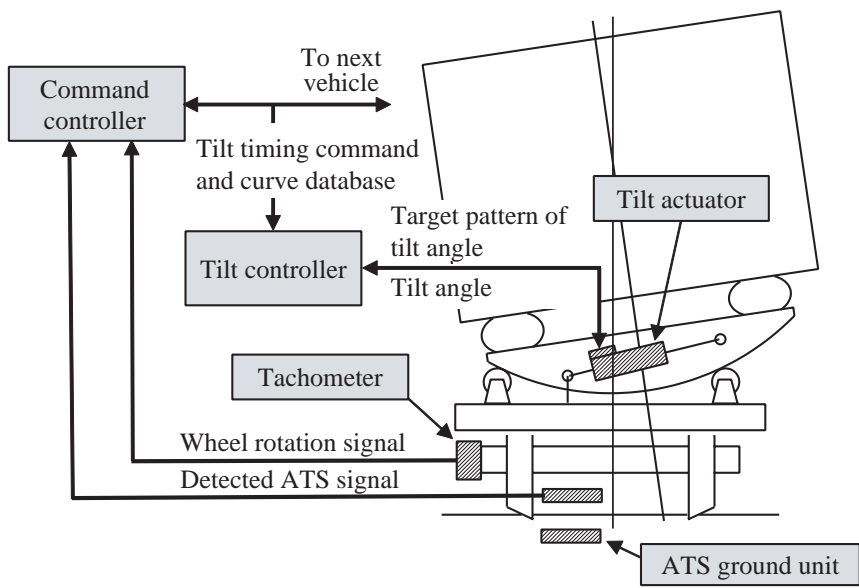

Fig. 1 Conventional tilt control system
Table 1 Resonant frequency of vehicle with conventional tilt control system

\begin{tabular}{c|c}
\hline Vehicle body vibration & $\begin{array}{c}\text { Resonant frequency } \\
{[\mathrm{Hz}]}\end{array}$ \\
\hline Lateral movement caused by air spring & 0.65 \\
\hline Rolling movement caused by air spring & 0.56 \\
\hline Yawing movement caused by air spring & 0.89 \\
\hline Rolling movement caused by tilt & 0.27 \\
\hline $\begin{array}{c}\text { Rolling movement caused by } \\
\text { pneumatic tilt actuator }\end{array}$ & 0.22 \\
\hline
\end{tabular}

the curve geometry. Since passive tilting, which is caused by unbalanced centrifugal forces to vehicle body, lags behind the geometry of the curves, the tilt actuator compensates for this.

It was envisaged that these vehicles would be able to run at high speeds and provide sufficient riding comfort. However, a survey of the actual conditions reveals the fact that some passengers in these trains are suffered from train motion sickness. As a result, it has been ascertained that the vibration that chiefly affects train motion sickness is lateral motion in the $0.25 \mathrm{~Hz}$ to $0.32 \mathrm{~Hz}$ frequency range ${ }^{1)}$. Table 1 sets out the resonant frequencies of a vehicle fitted with the conventional tilt control system. The resonant frequency caused by air springs is the same as that of a non-tilting vehicle, whereas the body rolling frequency caused by the tilt and pneumatic tilt actuators 
is peculiar to that of vehicles with tilt control. As rolling vibration tends to cause train motion sickness among passengers, we developed the next-generation tilt control system with a view to reducing the lateral vibration.

\section{Next-generation tilt control system}

As shown in Fig. 2, the next-generation tilt control system mainly consists of the following three newly developed technologies: position detection, tilting angle target pattern, and a novel tilt actuator. Firstly, position detection utilizes GPS signals, curvature collation and the wheel rotation speed in combination ${ }^{2) 3}$. Secondly, the tilting angle target pattern is expressed as a function of time and named the JT pattern (JT: Judgment function with $\mathrm{TC}_{\mathrm{T}}$ ). The $\mathrm{TC}_{\mathrm{T}}$ is an evaluation index that shows riding comfort in a transition curve section. Lastly, we developed a new electro-hydraulic actuator (EHA) to realize the JT pattern. Fig. 3 shows the total composition of the system.

Moreover, we have designed the new tilt system so that it works as a passive tilting system, because this will allow a vehicle to maintain its present speed even when the tilt control system breaks down. However, riding comfort in this case will deteriorate compared with normal system operation.



Fig. 2 Three technical elements

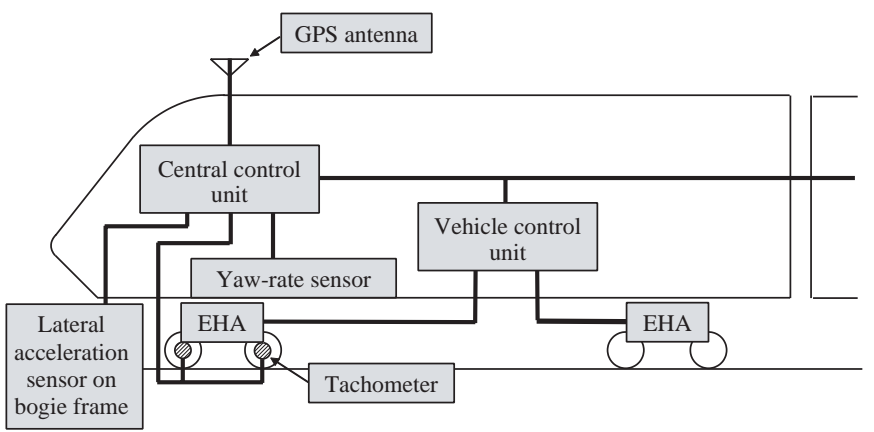

Fig. 3 Total composition of next-generation tilt control system

\section{Tilt actuator}

We investigated the type of drive that could be applied to the tilt actuator. Available technologies are the pneumatic, hydraulic, electro-mechanical or electro-hydraulic type. Table 2 compares characteristics for each type.
Table 2 Comparison of tilt actuator characteristics $\bigcirc$ Good $\triangle$ Normal $\times$ Not satisfactory

\begin{tabular}{c|c|c|c|c|c|c}
\hline $\begin{array}{c}\text { Type of } \\
\text { drive }\end{array}$ & Response & $\begin{array}{c}\text { Damper } \\
\text { function }\end{array}$ & Backforce & $\begin{array}{c}\text { Mass and } \\
\text { size }\end{array}$ & $\begin{array}{c}\text { Number } \\
\text { of parts }\end{array}$ & Maintenance \\
\hline Pneumatic & $\times$ & $\times$ & $\bigcirc$ & $\Delta$ & $\Delta$ & $\bigcirc$ \\
\hline Hydraulic & $\bigcirc$ & $\bigcirc$ & $\bigcirc$ & $\bigcirc$ & $\times$ & $\times$ \\
\hline EMA $^{*}$ & $\bigcirc$ & $\times$ & $\times$ & $\triangle$ & $\bigcirc$ & $\triangle$ \\
\hline EHA & $\bigcirc$ & $\bigcirc$ & $\bigcirc$ & $\triangle$ & $\bigcirc$ & $\bigcirc$ \\
\hline
\end{tabular}

The hydraulic type responds quickly and has a smaller, lighter cylinder than other types. However, it has many components, for instance, piping to connect it to a hydraulic pressure source and its operating oil must be protected from contamination.

The electro-mechanical type consists of a servo motor and a ball or planet roller screw. This actuator has quick response characteristics as well as high energy efficiency and can be easily handled to be connected to other devices simply by electric wires. However, its backforce is large against passive tilting operation.

In the case of the electro-hydraulic actuator (EHA), its servo motor, accumulator, pump and valve unit are unified with the cylinder, as shown in Fig. 4. Fig. 5 demonstrates the EHA's hydraulic oil circuit that includes

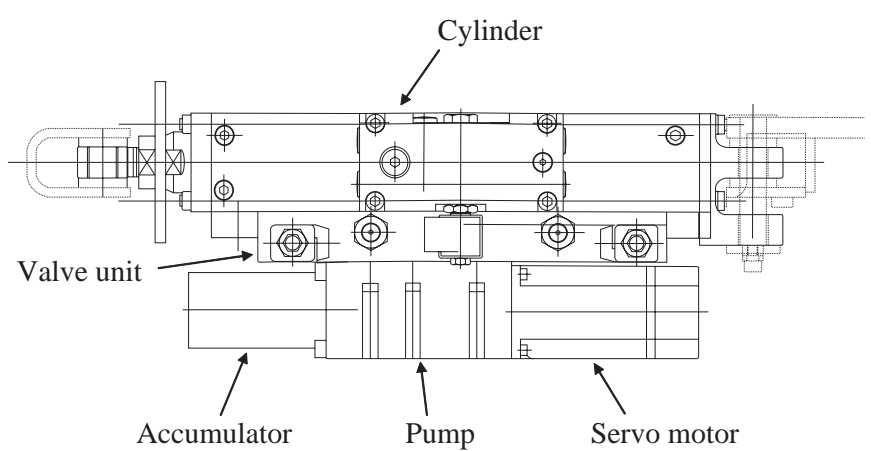

Fig. 4 Electro-hydraulic actuator (EHA) structure

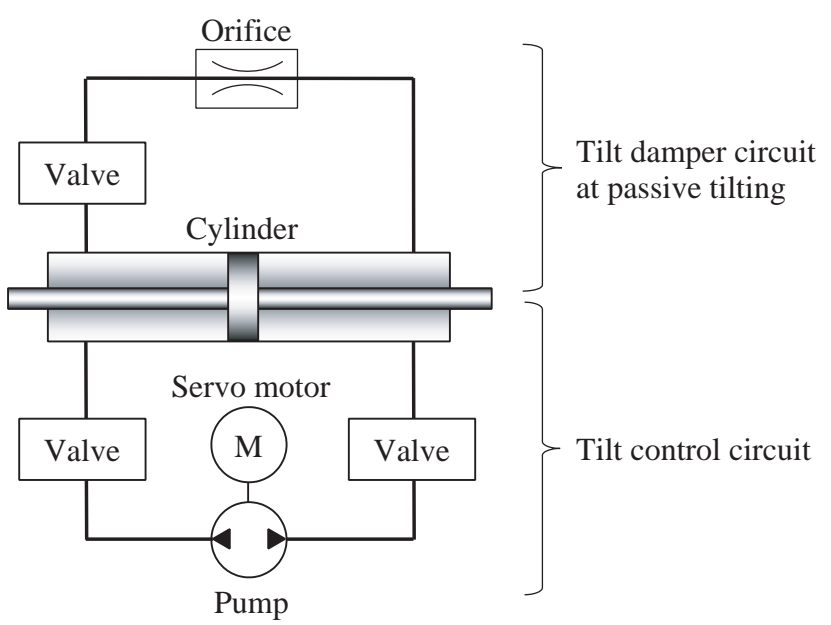

Fig. 5 EHA hydraulic pressure circuit 
Table 3 EHA specifications

\begin{tabular}{c|c}
\hline Rating drive force & $4 \mathrm{kN}$ at $100 \mathrm{~mm} / \mathrm{s}$ \\
\hline Maximum drive force & $9.8 \mathrm{kN}$ \\
\hline Maximum driving speed & $100 \mathrm{~mm} / \mathrm{s}$ \\
\hline Position precision & $\pm 1 \mathrm{~mm}$ \\
\hline Maximum pressure & $14 \mathrm{MPa}$ \\
\hline Damping coefficient & $20 \mathrm{Ns} / \mathrm{mm}$ \\
\hline Installation length & $750 \mathrm{~mm}$ \\
\hline Maximum stroke & $\pm 150 \mathrm{~mm}$ \\
\hline Power supply & $\mathrm{AC} 200 \mathrm{~V}$ \\
\hline
\end{tabular}

dual circuits, one for controlling and the other for damping the tilting movement. The EHA is driven according to the number and the direction of the servo motor rotations during tilt control. During passive tilting, the mounted orifice acts as a damper.

The above review shows that the EHA is the most suitable for a tilt actuator. Table 3 shows the specification of the designed EHA.

\section{Target pattern of tilting angle}

The JT pattern can be computed based on the current train speed and the onboard track geometry database so that the riding comfort index may be optimized.

\subsection{Track geometry database}

The onboard track geometry database consists of curvature and cant values actually measured discretely ev-
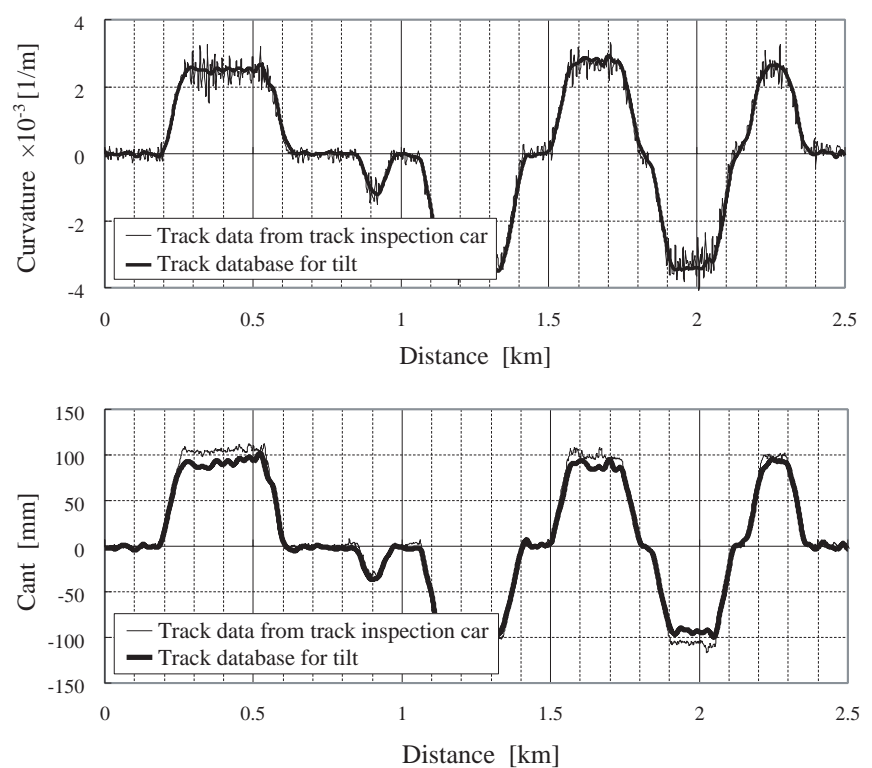

Fig. 6 Track geometry database ery $1 \mathrm{~m}$ length of a section. As illustrated in Fig. 6, there is a high coincidence between the curvature values in the database and those measured by a track inspection car, whereas the database cant values are slightly smaller than those by the inspection car. This is because the sensor used to draw up the database was attached on a bogie frame and could not measure the roll angle caused by the primary suspension. Since the EHA tilts a vehicle body at the required angle against a bogie frame, that causes no serious problem on tilt control.

\subsection{Riding comfort evaluation index}

We decided to compute the JT pattern using the $T C_{T}$ index ${ }^{4)}$, which evaluates the riding comfort in a transition curve section. This index is expressed as the sum of four weighted accelerations and those rates of a vehicle body, which are

$$
\begin{array}{r}
T C_{T-R}=0.6 Y_{p}+0.3 Y_{j}+0.03 \Theta_{p}+0.12 \Theta_{j}+0.5 \\
\text { for standing passengers, } \\
T C_{T-Z}=0.4 Y_{p}+0.4 Y_{j}+0.02 \Theta_{p}+0.04 \Theta_{j}+0.8 \\
\text { for seated passengers, }
\end{array}
$$

where $Y_{p}$ is the maximum lateral acceleration, $Y_{j}$ is the maximum rate of change in lateral acceleration (jerk), $\Theta_{p}$ is the maximum roll angular velocity, and $\Theta_{j}$ is the maximum rate of change in roll angular velocity.

The JT pattern is computed with the equation for $T C_{T \text { - }}$ ${ }_{R}$ standing passengers above.

\subsection{JT pattern generation}

The JT pattern is generated by the following procedure, as shown schematically in Fig. 7:

(1) The target pattern of tilting angle corresponding to time $0 \leq t \leq T_{0}$ is generated.

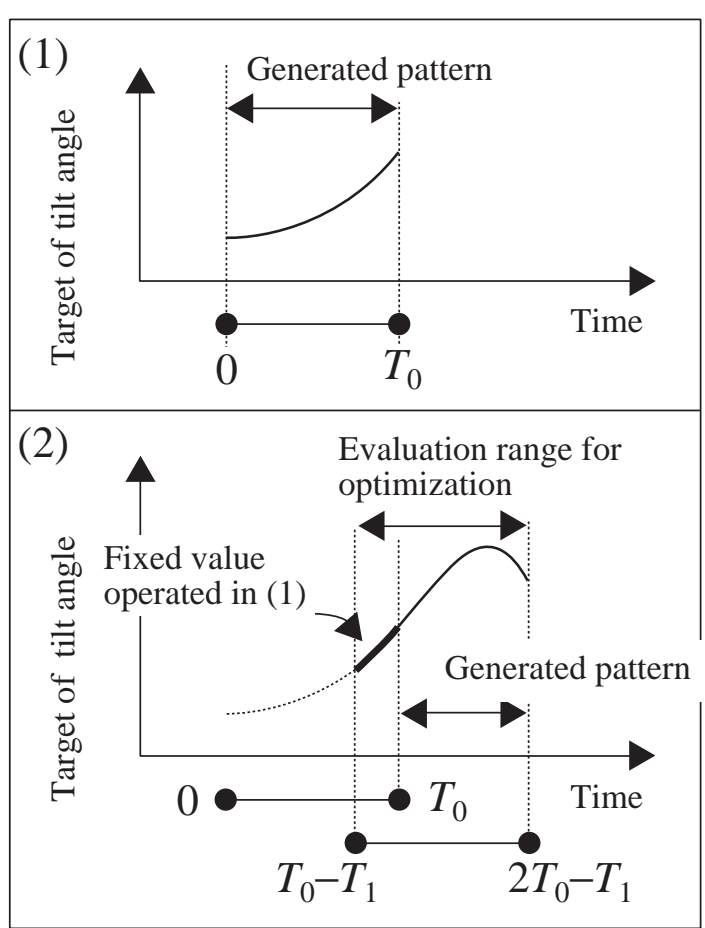

Fig. 7 JT pattern generation procedure 
(2) The next target pattern corresponding to time $T_{0} \leq t$ $\leq 2 T_{0}-T_{1}$ (where $0 \leq T_{1} \leq T_{0}$ ) is generated before the time reaches $T_{0}$.

(3) Thereafter the target pattern corresponding to the time duration $T_{0}-T_{1}$ is repeatedly added.

The computation for generating the JT pattern is described in detail as follows. The evaluation index of the riding comfort when the tilting angle pattern $\phi(t)$ for $0 \leq t$ $\leq T_{0}$ is predicted by the following equation,

$0.6 \max \left|y_{p}(t)\right|_{0}^{T_{0}}+0.3 \max \left|y_{j}(t)\right|_{0}^{T_{0}}+0.03 \max \left|\theta_{p}(t)\right|_{0}^{T_{0}}+0.12 \max \left|\theta_{j}(t)\right|_{0}^{T_{0}}$

where

$\max |x(t)|_{0}^{T_{0}}$ shows the maximum value of $x(t)$ for $0 \leq t \leq T_{0}$, $y_{p}(t)\left[\mathrm{m} / \mathrm{s}^{2}\right]$ is the lateral acceleration of a vehicle body,

$y_{j}(t)\left[\mathrm{m} / \mathrm{s}^{3}\right]$ is the differential of $y_{p}(t)$,

$\theta_{p}(t)[\%]$ is the roll angular velocity of a vehicle body, $\theta_{j}(t)\left[\% \mathrm{~s}^{2}\right]$ is the differential of $\theta_{p}(t)$.

In order to express $y_{p}(t), y_{j}(t), \theta_{p}(t)$, and $\theta_{j}(t)$ in Eq. (1) as a function of $\phi(t)$, we express them as Eqs. (2) (5), assuming an unbalanced centrifugal force is semi-statically approximated:

$$
\begin{aligned}
& y_{p}=\frac{v^{2}}{R(t)}-\left(\frac{C(t)}{G}+\phi(t)\right) g \\
& y_{j}=\dot{y}_{p}=-\frac{v^{2}}{R(t)^{2}} \dot{R}(t)-\left(\frac{\dot{C}(t)}{G}+\dot{\phi}(t)\right) g \\
& \theta_{p}=\frac{\dot{C}(t)}{G}+\dot{\phi}(t) \\
& \theta_{j}=\dot{\theta}_{p}=\frac{\ddot{C}(t)}{G}+\ddot{\phi}(t)
\end{aligned}
$$

where

$1 / R(t)[1 / \mathrm{m}]$ is the curvature of the curve,

$C(t)[\mathrm{m}]$ is the cant of the curve,

$v[\mathrm{~m} / \mathrm{s}]$ is the vehicle velocity (assuming that the value of $v$ is fixed when $0 \leq t \leq T_{0}$ ),

$G[\mathrm{~m}]$ is the gauge,

$g\left[\mathrm{~m} / \mathrm{s}^{2}\right]$ is the acceleration of gravity.

By substituting Eqs. (2), (3), (4) and (5) into Eq. (1), we obtain the following equation that evaluates $\phi(t)$,

$$
\begin{aligned}
& 0.6 \max \left|y_{p}(\phi(t))\right|_{0}^{T_{0}}+0.3 \max \left|y_{j}(\phi(t))\right|_{0}^{T_{0}}+0.03 \max \left|\theta_{p}(\phi(t))\right|_{0}^{T_{0}} \\
& +0.12 \max \left|\theta_{j}(\phi(t))\right|_{0}^{T_{0}}
\end{aligned}
$$

The optimal tilting pattern is obtained as $\phi(t)$ that minimizes the evaluation function Eq. (6), the pattern that gives optimal riding comfort.

Furthermore, we add the following conditions to $\phi(t)$ for applying the obtained tilting pattern for actual control.

(a) The value of $\phi(t)$ should not exceed the tilt angle limits.

(b) The series of $\phi(t)$ values should be smoothly connected with the preceding pattern.

To satisfy condition (a), we added some constraints to Eq. (6) and to satisfy condition (b) shall overlap the adjacent evaluation sections. The tilting angle that minimizes the evaluation function Eq. (6) and satisfies the two con-

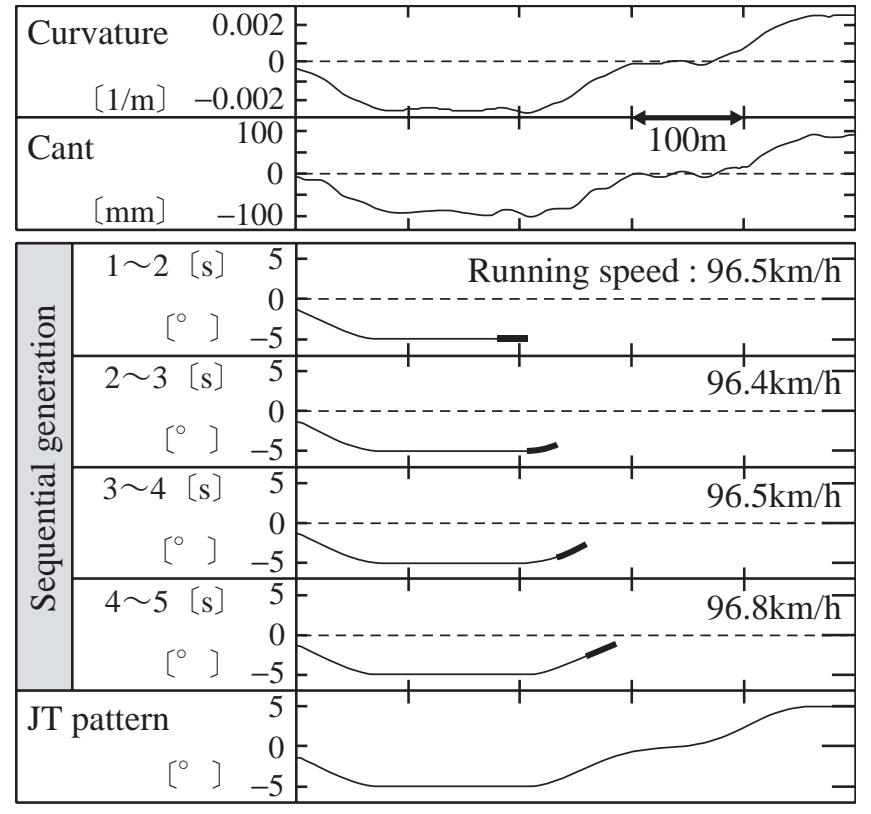

Fig. 8 Sequential generation of JT pattern

ditions mentioned above is named the JT pattern. Fig. 8 shows an example of a sequentially generated JT pattern.

\section{Result of running tests}

\subsection{Outline of running tests}

The tests were performed on an $11-\mathrm{km}$ section that included 28 curves with radius between $300 \mathrm{~m}$ and $800 \mathrm{~m}$. We compiled the databases for position detection and track geometry beforehand. Fig. 9 shows an EHA installed on a bogie. The measured items were position detection accuracy, the tilt operation response performance and riding comfort. As the position detection accuracy results were described in references 2 ), 3), we describe other two items on this paper.

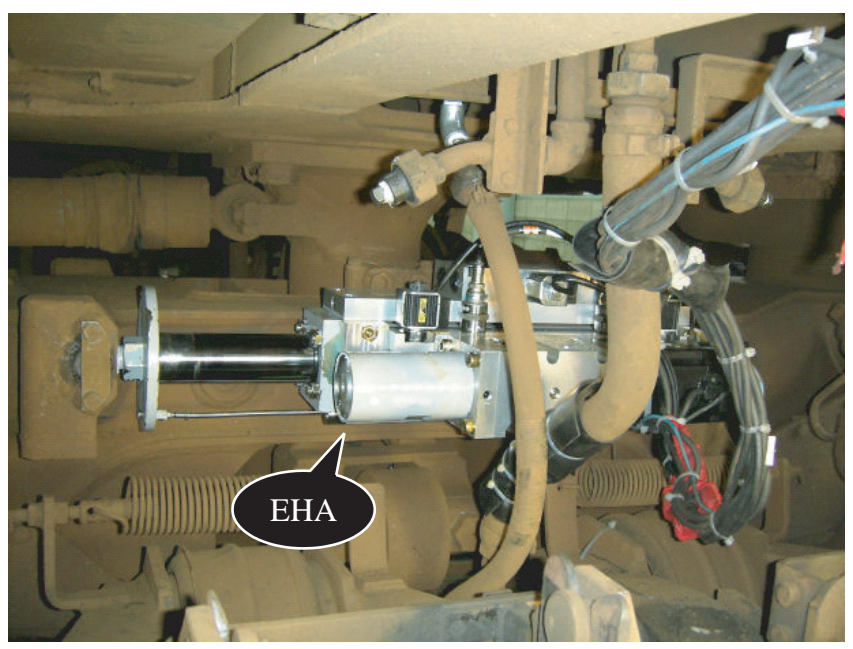

Fig. 9 EHA installed on bogie 


\subsection{Tilt operation response performance}

Fig. 10 shows the tilt angle, driving force and lateral acceleration of a vehicle body in curves obtained from both the novel EHA and a conventional pneumatic actuator. EHA response performance was very quick, and unstable low frequency vibration seen in the case of a pneumatic actuator did not occur. In the frequency range that causes motion sickness, the EHA's power spectrum density (PSD) for lateral acceleration was smaller than that of the pneumatic actuator, as shown in Fig. 11. On the other hand, the EHA displayed a larger PSD in frequency ranges higher than $0.9 \mathrm{~Hz}$. The reason for this is that the vibration absorption performance is decreased because an EHA's equivalent rigidity is larger. To counteract this, we are examining the installation of a vibration control device.
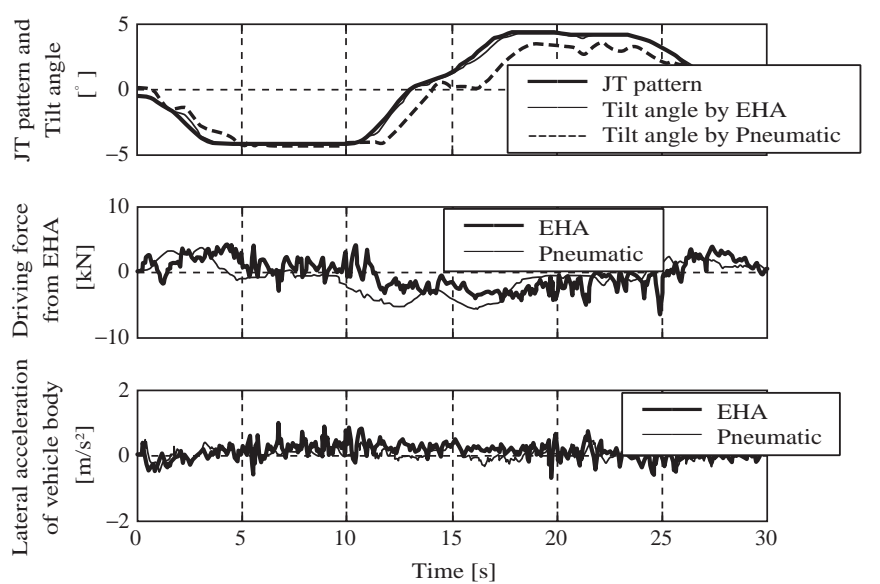

Fig. 10 Measured result in running test



Fig. 11 Power spectrum density for lateral acceleration of vehicle body

\subsection{Riding comfort evaluation}

(1) Results of passenger survey

A passenger survey in curve sections has been analyzed using the following four-grade scale:

1: I felt well

2: I felt slightly unwell

3: I felt unwell (riding comfort acceptable for a com- mercial train)

4: I felt unwell (riding comfort unacceptable for a commercial train)

As the result of the analysis, the average grade for the developed system was 1.4 against 1.6 for the conventional system. We concluded that the developed system's riding comfort offers an improvement over the conventional one.

(2) Motion sickness rates (MR)

MR shows the degree of motion sickness generation for passengers under lateral vibration. The index is calculated from the lateral acceleration through the filter for motion sickness on trains ${ }^{1)}$. The developed system's MR was 2.0 points lower than the average on the conventional system, confirming that this system decreases low frequency vibration.

(3) Riding quality level (Lt)

Lt is calculated from acceleration through the filter for vibration with the wide range frequency. It is evaluated using five grades ranging from "excellent" to "very poor". The developed system was graded "good," but it was larger by $1 \mathrm{~dB}$ to $2 \mathrm{~dB}$ in comparison with the conventional system. This may be due to the EHA's equivalent rigidity.

\section{Conclusions}

(1) To improve the performance and riding comfort of tilt control vehicles, we developed the next-generation tilt control system using a position detecting system, a tilting angle target pattern, a tilt actuator and other new technical elements.

(2) We developed the JT pattern based on the riding comfort evaluation index for a tilting angle target pattern and an electro-hydraulic actuator (EHA) to act as a tilt actuator. The result of running tests has shown that the EHA's response performance to the JT pattern is quick enough. It has been proven to be applicable to tilting vehicles.

(3) It has been evaluated that the developed system's riding comfort was superior to that of the conventional system, according to a passenger survey and the motion sickness rate. In addition, we were able to decrease low frequency vibration that causes motion sickness.

(4) The vibration absorption performance has decreased in a part of the frequency range, because of the EHA's large equivalent rigidity. We are examining the installation of a vibration control device.

\section{Acknowledgments}

We sincerely appreciate great cooperation on the running tests Shikoku Railway Company staffs.

\section{References}

1) Suzuki, H., Shiroto, H., Tezuka, K.: "Effects of Low Frequency Vibration on Train Motion Sickness," QR 
of RTRI, Vol. 46, No. 1, pp. 35-39, 2005

2) Maki, Y., Enomoto, M., Sasaki, K., Tsujino, S.: "A System to detect the Train Position using GPS and a Track Geometry Database," RTRI Report, Vol. 17, No. 4, pp. 11-16, 2003 (in Japanese)

3) Sasaki, K.: "Position Detection System Using GPS for
Carbody Tilt Control," QR of RTRI, Vol. 46, No. 2, pp. 73-77, 2005

4) Suzuki, H., Shiroto, H., Tanaka, A., Tezuka, K., Takai, H.: "Psychophysical Evaluation of Railway Vibrational Discomfort on Curved Sections," QR of RTRI, Vol. 41, No. 3, pp. 106-111, 2000 